\title{
Lost in translation: the gap between what we know and what we do about cardiovascular disease
}

\author{
An estimated one million Australians at high risk may not have been receiving \\ recommended preventive treatments
}

$\mathrm{W}$ e still seem disappointingly distant from getting it right with disease prevention in Australia. An analysis of data from the 2011-12 Australian National Health Measures Survey reported in this issue of the $M J A^{1}$ found that most respondents at high risk of cardiovascular disease (CVD) were not receiving the recommended combination of blood pressure-lowering and lipid-lowering medications. Applying these estimates to the general population, almost one million Australians at high risk may not have been receiving these treatments, although their use has long been recommended by the national peak body on the basis of level A evidence.

Perhaps the data are incorrect? While the response rate to the survey among those aged $45-74$ years $(47 \%)$ was lower than desirable, survey respondents would be expected to have better, not worse, treatment rates than non-respondents. It is also likely that self-reporting overestimates treatment levels. Perhaps the data reflect the prevalence of contraindications to recommended treatment? Again, this is unlikely: definite contraindications to lipid-lowering and blood pressure-lowering medications are very rare, and placebo-controlled trials have shown that only a small proportion of subjects taking statins experience symptomatic side effects. ${ }^{2}$

\section{What is going wrong?}

How much of the problem is related to physicians not prescribing medications in the first place, and how much is due to patients not continuing to use them in the long term? For people with an acute coronary syndrome, prescription rates at discharge have climbed steadily in recent years; the main challenge seems to be the steady drop-off in adherence in the months and years following the acute event. ${ }^{3}$ Both patient- and prescriber-related factors contribute to this drop-off. Patient-related factors include comorbidities, mental health problems, social context, and their perceptions of their illness; even those who have a heart attack underestimate their risk, and misperceptions are associated with the persistence of risk-elevating behaviours, including smoking and non-adherence to medication. ${ }^{4}$ The cost of drugs may play a bigger role in the Australian context than is recognised. ${ }^{5}$ Physicians may fail to recognise non-adherence or explain the need to continue therapy. Over-attribution of "side effects" to medication is also a

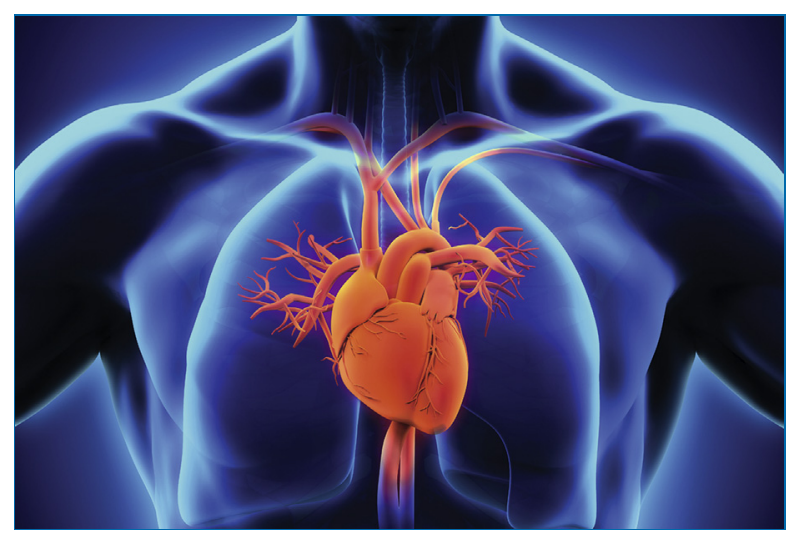

factor, ${ }^{2}$ compounded in Australia by biased media coverage about statins. ${ }^{6}$

\section{"diagnosis and treatment practices for}

hypertension remain similar to those of several decades ago"

For people at high primary risk of CVD, we suspect the main problem is under-prescribing of medications that reduce the risk of CVD. A paradigm change is needed, and we have only begun to address this need. The dominant paradigm for many decades has been to prescribe these medications only for people with hypertension or dyslipidaemia; indeed, many drug labels still recognise only these indications. The compelling rationale that use of these drugs should instead be primarily targeted on the basis of high absolute risk of CVD has been plain for many years. The evidence for the benefits of blood pressure-lowering medications for people with non-optimal blood pressure but without hypertension has become even clearer in the past few months. ${ }^{7}$ Nevertheless, diagnosis and treatment practices for hypertension remain similar to those of several decades ago, when it was erroneously thought there was a threshold of hypertension below which lowering blood pressure conferred no benefit.

\section{Do we need to try something new?}

We clearly need to improve our strategies for addressing the evidence-practice gap in CVD risk management. This is part of a wider systemic problem in continuing medical 
education, our ability to translate large amounts of new research data into practice.

Should we be considering a public health and policydriven approach? In the United Kingdom, the Quality and Outcomes Framework (QOF) annually rewards practices for their provision of quality care according to evidence-based indicators. ${ }^{8}$ For example, the indicators for the secondary prevention of coronary heart disease (CHD) include general practices maintaining a register of $\mathrm{CHD}$ patients and of the proportions prescribed angiotensin-converting enzyme inhibitors or angiotensin-receptor blockers, aspirin, $\beta$-blockers, and statins. ${ }^{8}$ A registry would enable practices to have annual recall programs for reviewing treatment of $C H D$ patients. In Australia, the National Health and Medical Research Council has recently adopted implementing the absolute risk approach to primary prevention as a priority for research translation. ${ }^{9}$

Technology-based solutions may also help. Hospital medical records, patient-controlled e-health medical records, and general practice medical records could incorporate red flags for pre-specified indicators of prevention needs not being met. Electronic decision support studies support the potential effectiveness of these type of strategies. ${ }^{10}$ Other technology-based solutions could include smart pill boxes, pharmacy support programs, telehealth support, and automated text reminders. ${ }^{11}$ These strategies are all ready to roll now, whereas other innovations, such as polypills, may play a role in the near future. ${ }^{12}$ There is evidence that supports the efficacy of all these strategies, but none has yet been substantively employed.

CVD is our leading killer and cause of health care costs, but almost one million Australians at high risk may not have been receiving the basic recommended preventive medications. This is the low-hanging fruit in CVD control. A multifaceted approach is needed: patient-centred, evidence-based primary care will be the key.

Acknowledgements: Clara Chow is funded by a Career Development Fellowship cofunded by the National Health and Medical Research Council (NHMRC) and the Heart Foundation, and by a Sydney Medical School Foundation Chapman Fellowship. Anthony Rodgers is funded by an NHMRC Principal Research Fellowship.

Competing interests: No relevant disclosures.

Provenance: Commissioned; externally peer reviewed.

(c) 2016 AMPCo Pty Ltd. Produced with Elsevier B.V. All rights reserved.

References are available online at www.mja.com.au. 
1 Banks E, Crouch SR, Korda RJ, et al. Absolute risk of cardiovascular disease events and blood pressure- and lipid-lowering treatment in Australia. Med J Aust 2016; 204: 320.el-320.e8.

2 Finegold JA, Manisty CH, Goldacre B, et al. What proportion of symptomatic side effects in patients taking statins are genuinely caused by the drug? Systematic review of randomized placebo-controlled trials to aid individual patient choice. Eur J Prev Cardiol 2014; 21: 464-474.

3 Redfern J, Hyun K, Chew DP, et al. Prescription of secondary prevention medications, lifestyle advice, and referral to rehabilitation among acute coronary syndrome inpatients: results from a large prospective audit in Australia and New Zealand. Heart 2014; 100: 1281-1288.

4 Thakkar J, Heeley EL, Chalmers J, Chow CK. Inaccurate risk perceptions contribute to treatment gaps in secondary prevention of cardiovascular disease. Intern Med J 2015; 46: 339-346.

5 Hynd A, Roughead EE, Preen DB, et al. The impact of copayment increases on dispensings of government-subsidised medicines in Australia. Pharmacoepidemiol Drug Saf 2008; 17: 1091-1099.

6 Schaffer AL, Buckley NA, Dobbins TA, et al. The crux of the matter: did the ABC's Catalyst program change statin use in Australia? Med J Aust 2015; 202: 591-595. https://www.mja. com.au/journal/2015/202/11/crux-matter-did-abcs-catalystprogram-change-statin-use-australia

7 Ettehad D, Emdin CA, Kiran A, et al. Blood pressure lowering for prevention of cardiovascular disease and death: a systematic review and meta-analysis. Lancet 2015; 387: 957-967.
8 NHS Employers. 2014/15 General Medical Services (GMS) contract quality and outcomes framework (QOF). Guidance for GMS contract 2014/15 (NHS England Gateway reference 01264). Mar 2014. http://www. nhsemployers.org/your-workforce/primary-care-contacts/ general-medical-services/quality-and-outcomes-framework (accessed Feb 2016).

9 Trevena L, Nelson M, Harris M, Osborne R. Case for Action proposal. The right care for the right patient at the right time: improving the identification and management of absolute cardiovascular risk in the community. 11 May 2015. http://www. nhmrc.gov.au/files_nhmrc/file/research/research_translation_ faculty/rtf_cfa_primary_health_care_150518.pdf (accessed Feb 2016).

10 Peiris D, Usherwood T, Panaretto K, et al. Effect of a computerguided, quality improvement program for cardiovascular disease risk management in primary health care: the treatment of cardiovascular risk using electronic decision support cluster-randomized trial. Circ Cardiovasc Qual Outcomes 2015; 8: 87-95.

1 Burke LE, Ma J, Azar KM, et al. Current science on consumer use of mobile health for cardiovascular disease prevention: a scientific statement from the American Heart Association. Circulation 2015; 132: 1157-1213.

12 Webster R, Patel A, Selak V, et al. Effectiveness of fixed dose combination medication ('polypills') compared with usual care in patients with cardiovascular disease or at high risk: a prospective, individual patient data metaanalysis of 3140 patients in six countries. Int J Cardiol 2016; 205: 147-156. 